\title{
Thermal fluctuations, mechanical response, and hyperuniformity in jammed solids
}

\author{
Atsushi Ikeda ${ }^{1}$ and Ludovic Berthier ${ }^{2}$ \\ ${ }^{1}$ Fukui Institute for Fundamental Chemistry, Kyoto University, Kyoto, Japan \\ ${ }^{2}$ Laboratoire Charles Coulomb, UMR 5221 CNRS-Université de Montpellier, Montpellier, France
}

(Dated: July 16, 2015)

\begin{abstract}
Jamming is a geometric phase transition occurring in dense particle systems in the absence of temperature. We use computer simulations to analyse the effect of thermal fluctuations on several signatures of the transition. We show that scaling laws for bulk and shear moduli only become relevant when thermal fluctuations are extremely small, and propose their relative ratio as a quantitative signature of jamming criticality. Despite the nonequilibrium nature of the transition, we find that thermally induced fluctuations and mechanical responses obey equilibrium fluctuation-dissipation relations near jamming, provided the appropriate fluctuating component of the particle displacements is analysed. This shows that mechanical moduli can be directly measured from particle positions in mechanically unperturbed packings, and suggests that the definition of a "nonequilibrium index" is unnecessary for amorphous materials. We find that fluctuations of particle displacements are spatially correlated, and define a transverse and a longitudinal correlation lengthscales which both diverge as the jamming transition is approached. We analyse the frozen component of density fluctuations and find that it displays signatures of nearly-hyperuniform behaviour at large lengthscales. This demonstrates that hyperuniformity in jammed packings is unrelated to a vanishing compressibility and explains why it appears remarkably robust against temperature and density variations. Differently from jamming criticality, obstacles preventing the observation of hyperuniformity in colloidal systems do not originate from thermal fluctuations.
\end{abstract}

PACS numbers: 05.10.-a, 05.20.Jj, 64.70.qj

\section{INTRODUCTION}

A jamming transition [1-3] occurs when it becomes too difficult to compress any further a dense assembly of hard objects, whose compressibility then vanishes. Remarkably, the same transition also controls the loss of mechanical rigidity observed when soft particles are decompressed in the absence of thermal fluctuations, such as foams or emulsion droplets, whose bulk modulus then vanishes. In both cases, the key variable controlling the physics is the particle connectivity, the jamming transition corresponding to the isostatic situation where just enough contacts are present to insure mechanical stability [4 7 ]. The distance to isostaticity controls the divergence of mechanical moduli observed when compressing hard particles and their vanishing when decompressing soft particles [7, 8]. The deep connection between geometry and mechanical responses shows that the criticality observed in the vicinity of the transition follows from the unambiguous identification of particle contacts. Therefore, when thermal fluctuations are present, for instance when considering colloidal particles (such as microgels, emulsions, PMMA colloids), the contacts can be 'blurred' by thermal agitation and cannot be resolved, which challenges the possibility to observe jamming criticality in experiments. In other words, a thermal system cannot know how far it is from isostaticity, and the associated criticality is easily destroyed by temperature [11].

In previous work, the role of thermal fluctuations near jamming has been explored to understand the influence of finite temperatures on various physical quantities such as microscopic dynamics, microstructure, contact number, mechanical properties $[920]$. In particular, a computer study of the single particle dynamics revealed the existence of a very narrow region in the (density, temperature) phase diagram where jamming criticality can be observed, which excludes most colloidal studies to date 11] - More recent experiments have concentrated on collective static properties, such as mechanical shear and bulk moduli and structure factors, and the results were analysed using power laws that are valid, strictly speaking, for fully athermal systems [21]. To assess the validity of this description, one needs to extend the analysis of Ref. 11] to mechanical moduli to understand whether their critical behaviour is robust against thermal fluctuations. The first goal of our work is to analyse the effect of thermal fluctuations on mechanical moduli near jamming.

Another property characterizing jammed packings is their hyperuniformity, which was revealed by analysing the large-distance scaling of volume fraction fluctuations [22]. For monodisperse spherical particles of diameter $\sigma$, this reduces to studying the ordinary static structure factor, $S(k)$, whose low-wavevector behavior obeys a nontrivial, characteristic linear behaviour, $S(k \sigma \ll 1) \sim k$, which shows that density fluctuations are suppressed at large scale [22, 23]. This behavior has been observed numerically in particle packings prepared exactly at the jamming transition [22 26], and experimentally in athermal granular materials [25]. Experiments performed with colloidal particles appear challenging and report only very weak signs of hyperuniformity $27-29]$. A possible explanation could be that hyperuniform behaviour is blurred by thermal fluctuations, as are other signatures of the jamming transition. However, hyperuniformity is a property of the packings at large lengthscale and the 
above argument regarding the resolution of particle contacts is not obviously relevant. Therefore, if hyperuniformity were affected by thermal fluctuations acting at the (vanishingly small) contact lengthscale, it would directly establish that hyperuniformity is another critical property associated to the jamming transition. The second goal of our work is to test whether hyperuniformity is robust against thermal fluctuations, and, more fundamentally, whether hyperuniformity is deeply related to the jamming transition, or is instead a distinct phenomenon.

Thermal fluctuations in jammed packings not only raise practical issues about experimental observations, they also pose fundamental challenges related to the nonequilibrium nature of the jamming transition. As mentioned above, mechanical moduli display power law behaviour near jamming at zero temperature. However, for materials at thermal equilibrium, mechanical response functions are directly related to mechanical fluctuations induced by thermal motion and thus to equilibrium structure factors through fluctuation-dissipation relations [30, 31]. Near the nonequilibrium jamming transition at finite temperatures, two behaviors are then possible:

1) Fluctuations and responses do not obey equilibrium relations, so that mechanical moduli and structure factors have independent density and temperature dependences. This hypothesis suggests that it could be useful to introduce novel variables to quantify deviations from equilibrium relations, such as nonequilibrium index [32, 33] or effective temperatures [34, 35], generically defined as ratios between fluctuations and responses. In that case, structure factors live an independent life from mechanical responses, and they may display an independent set of critical properties, but they may also have unremarkable behaviour near jamming. This general hypothesis has been advocated in particular in Refs. 32, 33], where a diverging nonequilibrium index and a diverging nonequilibrium lengthscale were defined from fluctuations and responses of hard sphere systems approaching jamming [32], and later extended to generic amorphous solids [33].

2) Fluctuations and responses obey equilibrium relations, and the critical behaviour of mechanical moduli should have a counterpart in fluctuations of particle positions and structure factors. In that case, if thermal fluctuations are finite (but still sufficiently small that they do not blur the jamming criticality!), interesting critical behaviour should be observed in density fluctuations of mechanically unperturbed packings. In particular, one may expect the emergence of diverging lengthscales in collective structure factors of jammed materials.

The third goal of our work is to decide which of the two above scenarios is valid, and whether interesting lengthscales and nonequilibrium indicators emerge from the analysis of structure factors.

To achieve our three main goals, we use computer simulations of a simple model of soft harmonic particles 36] to analyse the influence of thermal fluctuations on me- chanical responses and structure factors in the vicinity of the jamming transition. Harmonic spheres are convenient because they allow studies of multiple, experimentally relevant, routes to jamming in the (density, temperature) phase diagram [7, 14, 36 39]. Therefore, a single model provides us with decisive answers to the three sets of questions mentioned above, that can be summarized as follows.

(i) We find that mechanical moduli are as sensitive to thermal fluctuations as single particle dynamics and their associated power law behaviour is not a good starting point to theoretically describe existing colloidal experiments.

(ii) By contrast, hyperuniformity is extremely robust to the addition of thermal perturbations, and even to changes in packing fraction, suggesting that it should in fact be far easier to observe in experiments than the jamming criticality, even though the present state of the literature suggests the opposite. We also conclude that hyperuniformity bears no deep relation to the jamming transition, and in particular we show that it is fully unrelated to the critical behavior of the mechanical compressibility.

(iii) Equilibrium fluctuation-dissipation relations are perfectly obeyed near jamming, suggesting it is unnecessary to define quantitative indicators for the degree of 'nonequilibriumness' near jamming. It also implies that structure factors display critical properties and reveal diverging lengthscales, that we define, analyse, and compare to previously studied critical lengthscales.

This article is organised as follows. In Sec. [I] we define the model and our numerical strategy. In Sec. III we analyse the behaviour of mechanical moduli. In Sec. IV we define and analyse the behavior of structure factors and their associated lengthscales. In Sec. $\nabla$ we discuss the hyperuniformity of jammed packings. In Sec. VI we summarize and discuss our results.

\section{MODEL AND SIMULATION}

We consider a system of monodisperse harmonic spheres, interacting through a pairwise potential [36],

$$
v\left(r_{i j}\right)=\frac{\epsilon}{2}\left(1-r_{i j} / \sigma\right)^{2} \Theta\left(\sigma-r_{i j}\right),
$$

where $\Theta(x)$ is the Heaviside function, $r_{i j}$ is the distance between particles $i$ and $j$, and $\sigma$ is the particle diameter. Throughout this work, length, energy, temperature and mechanical moduli are measured in units of $\sigma, \epsilon, \epsilon / k_{B}$, and $\epsilon / \sigma^{3}$, respectively.

We use molecular dynamics simulations [40] to compute the mechanical moduli and static structure factors of the system at finite temperature. The setting of the calculations is essentially similar to our previous work [1]. We first generate a random configuration of $N=64,000$ particles in a simulation box with periodic boundary conditions. The linear dimension of the 
box $L$ is adjusted to realize the packing fraction fraction $\varphi=0.80$, where $\varphi=\pi \sigma^{3} N /\left(6 L^{3}\right)$. Starting from this configuration, we perform molecular dynamics simulations at $T=10^{-5}$, where we integrate Newton's equations of motion using velocity rescaling to control the temperature. This can be seen as an extensive aging of the system starting from $T=\infty$ down to $T=10^{-5}$. We find that temperature is low enough that aging dynamics eventually stops and the energy and average particle positions reach well-defined values that do not depend on waiting time any longer. After this long annealing of the system, we change the density and temperature to the desired values smoothly, letting the system relax at each state point before taking any measurement. This protocol allows us to study essentially the same particle packing at different densities and temperatures. We do not study temperatures larger than $T=10^{-5}$, and in the studied regime particle diffusion and rearrangements can be safely neglected.

At each state point, we then perform molecular dynamics simulations to calculate the mechanical moduli and static structure factors, where we integrate Newton's equations of motion in the $N V E$ ensemble, i.e. without thermostat. We denote the long-time average in these calculations with brackets, $\langle\cdots\rangle$. For the particular configuration which is analysed extensively in this work, the jamming density is $\varphi_{J} \simeq 0.648$.

For the specific purposes of Sec. V the static structure factor of jammed harmonic spheres with a larger number of particles, $N=512,000$, at strictly zero temperature. To this end, we generate a random configuration of particles in a simulation box with $\varphi=0.80$, and then apply the FIRE algorithm to minimize the potential energy of the system at this density [41]. Starting from this jammed configuration, we decrease the density by small steps and minimize the potential energy after each step to obtain a series of jammed particle configurations over a range of densities [7]. To increase the statistics of the results obtained for these zero-temperature packings, we followed this procedure starting from 8 independent random configurations, and finally averaged the results over these independent runs. For this series of simulations, we find that $\varphi_{J} \simeq 0.64571 \pm 0.00012$, where the errorbar indicates the standard deviation among independent packings. Averaging over those configurations is therefore accurate as long as the distance to the jamming density is larger than $\left|\varphi-\varphi_{J}\right| \approx 0.0001$.

\section{MECHANICAL MODULI AND JAMMING CRITICALITY}

In this section we analyse the temperature and density dependences of bulk and shear moduli of harmonic spheres in the vicinity of the jamming transition occurring at $\left(T=0, \varphi=\varphi_{J}\right)$.

\section{A. Bulk modulus}

We start our analysis with the calculation of the bulk modulus. The isothermal bulk modulus $B$ quantifies the resistance of the system to compression. Its definition then naturally involves the pressure (noted $P$ ) derivative of the volume (noted $V$ ),

$$
B=-V\left(\frac{\partial P}{\partial V}\right)_{T}
$$

We first calculate $B$ through the response formula Eq. (2), where the pressure $P$ is calculated from the virial formula

$$
P=\rho T+\frac{\langle W\rangle}{V},
$$

where $W=\sum_{i j} r_{i j} v^{\prime}\left(r_{i j}\right) / 3$ is the virial [31]. The bulk modulus is of course inversely proportional to the isothermal compressibility, $B=1 / \chi_{T}$. In practice we measure the pressure for various densities, and estimate the derivative in Eq. (2) using finite differences, which suggests that compressions or decompressions yield the same results. The numerical results are shown with open symbols and dashed lines in Fig. 1 .

The density dependence of the bulk modulus strongly depends on the temperature. At lower temperature, e.g. $T=10^{-8}$, the bulk modulus increases very sharply with density when $\varphi<\varphi_{J}$, and becomes essentially densityindependent when $\varphi>\varphi_{J}$. This behavior can be understood as a smooth crossover between the jamming of Brownian hard spheres and the unjamming of nonBrownian soft spheres. For $\varphi<\varphi_{J}$ and $T \rightarrow 0$, the particles have vanishing overlaps and essentially explore hard sphere configurations. The pressure of hard spheres diverges at the jamming transition as $P \sim T\left(\varphi_{J}-\varphi\right)^{-1}$, and as a result the bulk modulus behaves as [42]

$$
B \sim T\left(\varphi_{J}-\varphi\right)^{-2},
$$

which underlies both the critical nature of the transition and the entropic origin of solidity in hard particle systems. Our numerical results for $\varphi<\varphi_{J}$ can be well fitted with this power-law divergence. Equivalently, Eq. (4) implies that the isothermal compressibility $\chi_{T}$ vanishes quadratically with $\left(\varphi_{J}-\varphi\right)$ in this regime.

On the other hand, for $\varphi>\varphi_{J}$ at lower temperatures, particles may have finite overlaps, and thermal fluctuations play a small role. Thus, the system corresponds to non-Brownian soft spheres. For this system, the pressure emerges continuously at the jamming transition, $P \sim\left(\varphi-\varphi_{J}\right)$, and thus the bulk modulus is expected to be 7]

$$
B \sim \text { const. }
$$

Again, this behavior is in good agreement with the results shown in Fig. 1. 


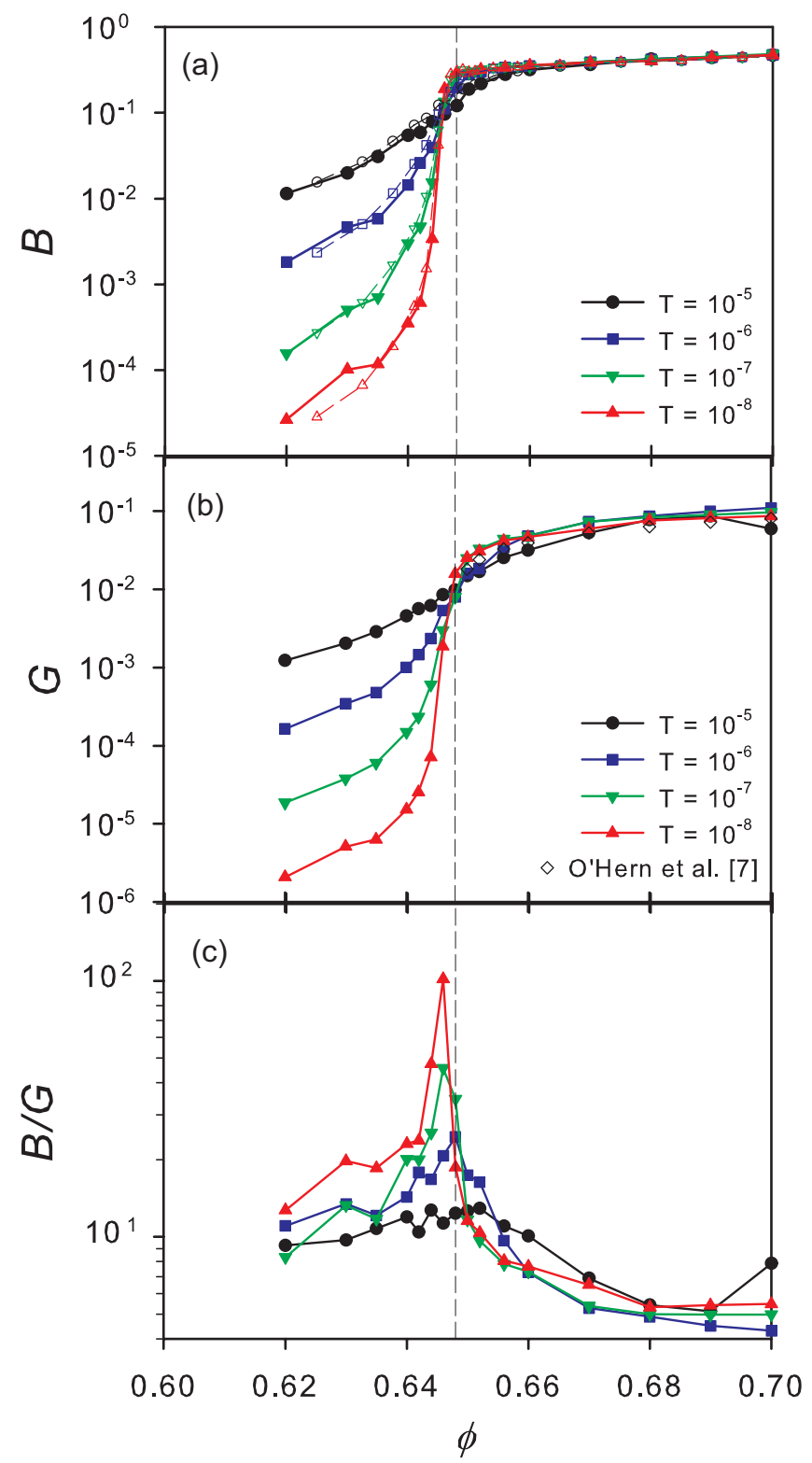

FIG. 1: (Color online) Density dependence of various quantities for different temperatures indicated in the label. (a) Bulk modulus. Results from the pressure derivative, Eq. (2), are shown with open symbols and dashed lines, results from the fluctuation formula, Eq. (6), are shown with filled symbols and solid lines. (b) Shear modulus obtained from Eq. (7). Open diamonds indicate data for $T=0$ obtained from response functions [7]. (c) Ratio of the bulk to shear modulus. The vertical dashed line indicates the location of the $T=0$ jamming transition at $\varphi_{J} \simeq 0.648$.

When temperature is increased above $T=10^{-8}$, the difference of behaviour observed on both sides of the transitions becomes smaller, and for the largest studied temperature, $T=10^{-5}$, the bulk modulus is a smooth function of density across $\varphi_{J}$. This shows that systems characterized by $T=10^{-5}$ (in units of the particle softness $\epsilon$ [15]) are unable to reveal signs of the underlying jamming transition at $T=0$. In particular, the solid behaviour of these systems is better interpreted as resulting from hitting a glass transition line $T_{g}(\varphi)$ [43]. This qualitative behavior is consistent with our previous discussion of the single particle dynamics [11].

We now use a different approach to compute the bulk modulus which does not involve a response function, but stems instead from studying thermal fluctuations in a mechanically unperturbed material. Within the framework of equilibrium statistical mechanics, the bulk modulus can be directly related to the fluctuations of the pressure. In the $N V E$ ensemble that we use to compute the pressure fluctuations, the formula for the isothermal bulk modulus reads [40]

$$
B=P+\frac{\left\langle W_{2}\right\rangle}{V}-\frac{\left\langle P^{2}\right\rangle-\langle P\rangle^{2}}{T} V+\frac{2}{3} \rho T-\frac{T \gamma_{V}^{2}}{\rho c_{V}},
$$

where $W_{2}=\sum_{i j}\left(r_{i j} v^{\prime}\left(r_{i j}\right)+r_{i j}^{2} v^{\prime \prime}\left(r_{i j}\right)\right) / 9$ is the hypervirial, $c_{V}$ is the specific heat per particle, and $\gamma_{V}$ is the thermal pressure coefficient [40]. The last term in Eq. (6) arises because we work in the microcanonical ensemble where the energy is conserved.

We have calculated the bulk modulus through Eq. (6), and report the results as filled symbols in Fig. 1. Clearly, the results are in excellent agreement with the ones obtained from the response formula, Eq. (2). Therefore, we conclude that fluctuations and response functions yield identical results for repulsive colloidal particles near jamming over a broad range of densities and temperatures. The natural interpretation is that equilibrium relations appear satisfied because deeply jammed solids dynamically explore a restricted portion of the configurational space located near a metastable amorphous configuration. In other words, the system is locally in equilibrium, even though ergodicity is globally broken. Using the language of the two-temperature scenario for aging glasses [34, the thermal fluctuations that are probed in the present system correspond to the fast degrees of freedom that appear locally equilibrated at the temperature of the thermal bath. This physical perspective justifies why it is unnecessary to introduce an effective temperature for the slow degrees of freedom, because these are completely frozen in the type of analysis that we perform. In other words, vibrational motion does not reveal the nonequilibrium nature of the glass.

Our results seem to contradict previous work [32] introducing a nonequilibrium index $X$ to quantify deviation from equilibrium behaviour between the bulk modulus $B$ (measured as a reponse function) and the smallwavevector limit of the static structure factor, $S(k \rightarrow 0)$, which reduces to the isothermal compressibility $\rho T \chi_{T}$ for equilibrium fluids. In Sec. IV] we clarify the relation between structure factors, pressure fluctuations, and compressibility, and show that the physical content of the "nonequilibrium" index introduced in Ref. [32] can in fact be fully understood in terms of equilibrium fluctuation-dissipation relations. 


\section{B. Shear modulus}

We now turn to the analysis of the shear modulus, $G$. There are several ways to compute the shear modulus in numerical simulations [40]. The first option is to use global fluctuations. Just as the bulk modulus can be determined from the fluctuations of the pressure, the shear modulus can be obtained from the fluctuations of the shear stress. We have first tried to use this approach to calculate the shear modulus, but found that an accurate determination of $G$ is not easy because the fluctuation formula requires to take the difference between large numbers which largely cancel and have important statistical fluctuations.

To overcome this problem, we use the alternative method introduced in Ref. [44]. In this approach, the shear modulus is calculated as the $k \rightarrow 0$ limit of the the correlation function of the transverse displacement $S_{T}(k)$,

$$
G=\lim _{k \rightarrow 0} \frac{\rho T}{S_{T}(k)} .
$$

The precise definition and detailed analysis of $S_{T}(k)$ will be given in Sec. IV] see Eq. (21). For the moment, we simply notice that the determination of $G$ from Eq. (7) clearly stems from spontaneous fluctuations, and this approach thus differs from earlier determinations based on response functions [7]. Here, we concentrate on the temperature and density dependences of the shear modulus $G$, and report our results in Fig. 1]. Note that this definition of the shear modulus does not require testing the validity of linear response, and does not depend either on the chosen direction for shearing. Although the shear modulus measured as a response function may depend on the direction of shear [45], all the directions of the shear modes are averaged out in the definition of $S_{T}(k)$ that we use in Eq. (21).

First, we check the validity of the fluctuation formula Eq. (77). To this end we compare our results to the shear modulus obtained from the response function in Fig. 1. Although the available data is limited to the density above $\varphi_{J}$ at $T=0$ [7], our results at lower temperature are quantitatively the same as the data from the response function at $T=0$. This confirms that fluctuations and response functions yield identical results for the shear modulus as well. A similar agreement between response and correlations for the shear modulus was reported in other glassy systems [44, 46], which appears as a robust result.

The overall behavior of the shear modulus is qualitatively similar to the one of the bulk modulus. At lower temperature, the shear modulus also increases very sharply with $\varphi$ below the jamming density, and has a more modest density dependence above jamming. A closer look to the numerical data indicates that the density dependence of $G$ is more pronounced above jamming than the one of $B$. Similarly to $B$, the sharp features of the shear modulus disappear rapidly when temperature is increased above $T=10^{-8}$, and again the density dependence is very smooth when $T \gtrsim 10^{-6}$. This indicates that the characteristic critical laws associated to the shear modulus near the jamming transition are easily smeared out by thermal fluctuations as well.

The low-temperature crossover behaviour observed for $G$ is again the signature of the zero-temperature criticality associated to the jamming transition. For $\varphi<\varphi_{J}$ and $T \rightarrow 0$, the system explores the divergence of the shear modulus of Brownian hard spheres approaching jamming, which follows

$$
G \sim T\left(\varphi_{J}-\varphi\right)^{-\kappa}
$$

where $\kappa \approx 1.41$ is a non-trivial critical exponent [16, 47, 48]. On the other side of the jamming transition, jammed harmonic spheres lose shear rigidity as the jamming density is approached from above [17],

$$
G \sim\left(\varphi-\varphi_{J}\right)^{1 / 2}
$$

Although our numerical results are consistent with Eqs. (8, 9), they are not precise enough to confirm that the exponent $\kappa$ is different from a previous estimate $\kappa=3 / 2$ [9], which is only marginally different from its recently predicted value $\kappa \approx 1.41$.

\section{Ratio $B / G$ of bulk to shear modulus: a signature of jamming criticality}

Whereas we noted that both $B$ and $G$ show qualitatively similar sharp features in the vicinity of the jamming transition, we also stated that the precise values of the exponents characterizing their power law behavior are different. These differences stem from the fact that the behaviour of the bulk modulus $B$ can be understood from the evolution of the pressure, whereas the behavior of $G$ is ruled by the evolution of the response of the system to a shear deformation. It is a specific signature of the jamming transition that responses to shear and to compression differ maximally for isostatic packings [1, 2, 7, 49, 50].

Therefore, to clearly detect a quantitative sign of the jamming criticality, it is useful to analyse the behavior of the ratio $B / G$, which becomes infinite at the critical point. We combine our finite temperature data for $B$ and $G$ to follow the density dependence of $B / G$ for various temperatures in Fig. 1 For our lowest temperature, $T=10^{-8}$, we find that $B / G$ is of order $5-10$ far from the jamming density, but has a sharp maximum of or$\operatorname{der} B / G \approx 100$ when $\varphi \approx \varphi_{J}$. This behavior should be interpreted as a smooth version of the zero-temperature density dependence, which follows from Eqs. (4, 5, 8, 9):

$$
\begin{aligned}
B / G & \sim\left(\varphi_{J}-\varphi\right)^{\kappa-2}, \quad \varphi<\varphi_{J} \\
& \sim\left(\varphi-\varphi_{J}\right)^{-1 / 2}, \quad \varphi>\varphi_{J}
\end{aligned}
$$


where $\kappa-2 \approx-0.59$. Notice that the behaviour of $B / G$ is now more symmetric around the jamming transition as the temperature prefactor disappears from the ratio $B / G$, but the critical exponents slightly differ on both sides of the transition (the divergence should be sharper for $\left.\varphi<\varphi_{J}\right)$. Note also that the behavior of $B / G$ is quantitatively analogous to the behavior of the adimensional mean-squared displacement defined in Ref. [11]. Therefore, this figure demonstrates that the impact of thermal fluctuations on mechanical moduli and on single particle dynamics is actually identical, and mechanical moduli are in fact equally fragile against Brownian motion.

We interpret the smoothened version of the symmetric divergence described by Eq. (11) observed for $T=10^{-8}$ in Fig. 1 as a 'thermal vestige' of the jamming transition [18], which is equivalent to the adimensional meansquared displacement defined in Ref. [11]. These two quantities are both direct signatures of jamming criticality and should therefore be contrasted with a nonmonotonic behaviour of the pair correlation functions [12, 18] which is instead a more general consequence of the particle softness and, as such, survives arbitrarily far from the critical point [51]. Therefore we suggest that the observation of a large $B / G$ ratio is a genuine sign that a particular material lies in the critical regime of the jamming transition, whereas a density-maximum in the pair correlation function is not.

When the temperature is increased, the non-monotonic density dependence of $B / G$ is rapidly erased by thermal fluctuations. For $T=10^{-5}$, we observe that $B / G$ is nearly independent of the density and has a value of order $5-10$ at all $\varphi$. These findings directly confirm that the jamming criticality is rapidly smeared out by thermal fluctuations. We also notice that even for very low temperatures, the range of densities where anomalous behavior associated to jamming can be observed is extremely narrow. These conclusions, obtained from the analysis of mechanical moduli, are in full agreement with previous conclusions drawn from the analysis of the mean-squared displacements [11].

In Ref. [21], the bulk and shear moduli of a twodimensional assembly of soft microgels were analysed, and their density evolution interpreted in terms of the power laws associated to the zero-temperature jamming criticality. Previous analysis of similar microgel systems has shown that these particles are quite soft, so that thermal fluctuations are of order $T \approx 10^{-6}-10^{-4}$, depending on the precise experimental system [15, 52]. The data reported in Ref. 21] show that the ratio $B / G$ is $B / G \approx 3$ with a very weak density dependence. This is very much consistent with the physical interpretation that this system is far from being critical, which reinforces the general conclusion that very soft microgel systems are not good experimental systems to reveal thermal vestiges of the jamming transition. In Ref. [15], we suggested that emulsion droplets might be better suited for this task, as recently confirmed experimentally [53].

\section{STATIC STRUCTURE FACTORS AND DIVERGING LENGTHSCALES}

In this section we define and study a number of static structure factors that can be probed in kinetically arrested colloidal materials in the presence of thermal fluctuations. From their low-wavevector analysis, we define lengthscales that diverge as the $T=0$ jamming transition is approached in the $(T, \varphi)$ plane.

\section{A. Definitions of structure factors}

Because we know the position of each particle in each configuration, we can define a number of static structure factors from our particle packings at finite temperatures.

The standard definition of the static structure factor is given by

$$
S(k)=\frac{1}{N}\left\langle\rho_{\vec{k}} \rho_{-\vec{k}}\right\rangle,
$$

where we have defined the Fourier transform of the density field as

$$
\rho_{\vec{k}}=\sum_{j} \exp \left(-i \vec{k} \cdot \vec{R}_{j}\right)
$$

with $\vec{R}_{j}$ the position of particle $j$. We assume that all our packings are isotropic so that structure factors only depend upon wavevectors through their moduli $k=|\vec{k}|$.

For a simple fluid at thermal equilibrium, the zero wavevector limit of $S(k)$ is directly related to the bulk modulus, and we have

$$
\lim _{k \rightarrow 0} S(k)=\frac{\rho T}{B} \quad \text { (Fluid). }
$$

This relation is only correct for liquid states 31]. We shall see below that a different analysis is needed for jammed solids.

We consider systems that are lacking crystalline order but are nevertheless kinetically arrested. This implies that translational invariance is actually broken, and that we consider instead materials with long-range "amorphous order" to use the language of glass theories [43, 54]. In practice, this means that the particle positions, and therefore, density fluctuations, can be naturally decomposed into two different contributions, from which two distinct structure factors can be defined:

$$
\begin{aligned}
S_{\delta}(k) & =\frac{1}{N}\left\langle\delta \rho_{\vec{k}} \delta \rho_{-\vec{k}}\right\rangle, \\
S_{0}(k) & =\frac{1}{N}\left\langle\rho_{\vec{k}}\right\rangle\left\langle\rho_{-\vec{k}}\right\rangle,
\end{aligned}
$$

with $\delta \rho_{\vec{k}}=\rho_{\vec{k}}-\left\langle\rho_{\vec{k}}\right\rangle$. From the definitions (12, 15, 16) it is obvious that

$$
S(k)=S_{\delta}(k)+S_{0}(k),
$$


showing that we have obtained a decomposition of the structure factor in terms of a fluctuating part, $S_{\delta}(k)$, and a configurational part, $S_{0}(k)$. Physically, $S_{0}(k)$ represents the structure factor associated to the averaged position of the particles and is essentially independent of temperature and weakly dependent of density; it is an 'inherent' property of the amorphous packing [55]. By contrast, $S_{\delta}(k)$ represents the structure factor associated to the fluctuations of the particles away from their average positions, and a strong temperature dependence is expected for this contribution, which should for instance vanish as $T \rightarrow 0$ for $\varphi>\varphi_{J}$, when particles stop moving completely.

When translational invariance is broken, as in crystals and glasses, the bulk modulus of the system is no longer related to $S(k)$ as in Eq. (14), but to the fluctuation part $S_{\delta}(k)$ [30]. Within a conventional conventional elasticity theory where the elastic moduli are assumed to be independent of wavevector, an elastic body is characterized by longitudinal plane waves with the dispersion relation $\omega=k \sqrt{\left(B+\frac{4}{3} G\right) / \rho}$. These plane waves are thermally excited and follow the equipartition law, so that the fluctuating part of the density fluctuations is given by 63 .

$$
S_{\delta}(k)=\frac{\rho T}{B+\frac{4}{3} G} \quad(\text { Continuum solid }) .
$$

This is the fluctuation formula appropriate for connecting a static structure factor to the bulk modulus in a solid state. It is obviously distinct from the formula valid for fluids, Eq. (18). Notice that the distinction between the two formula stems from the fact that translational invariance is broken (in solids) or not (in fluids), but both formula rely on the fact that the system (fluid or solid) obeys the rules of equilibrium thermodynamics.

It is also useful to provide a dynamic interpretation of the decomposition in Eq. (17). Because the dynamics is arrested and the system only probes thermal fluctuations near a given metastable state, the dynamic structure factor does not decay to zero at long times. The intermediate scattering function is $F(k, t)=\left\langle\rho_{\vec{k}}(0) \rho_{-\vec{k}}(t)\right\rangle / N$, so that $F(k, t=0)=S(k)$. In the long-time limit, density fluctuations are uncorrelated, and we directly find that $F(k, t \rightarrow \infty)=S_{0}(k)$, which is nothing but the collective Debye-Waller factor. Therefore, the fluctuation part of the static structure can be written as $S_{\delta}(k)=S(k)-F(k, t \rightarrow \infty)$, which quantifies the relaxing part of the density fluctuations.

In addition, we define two more structure factors associated to the particle positions, which rely on the vectorial character of the displacement field. In solid states, each particle vibrates around its average position. We can then define the displacement of each particle as $\vec{u}_{i}=\vec{R}_{i}-\left\langle\vec{R}_{i}\right\rangle$, and the associated displacement field, expressed in the Fourier domain as

$$
\vec{u}_{\vec{k}}=\sum_{j} \vec{u}_{j} \exp \left(-i \vec{k} \cdot\left\langle\vec{R}_{j}\right\rangle\right)
$$

In the Fourier space, we can then decompose the displacement field into its longitudinal and transverse parts:

$$
\vec{u}_{\vec{k}}=\hat{k} u_{L, \vec{k}}+\vec{u}_{T, \vec{k}}
$$

where $u_{L, \vec{k}}=\hat{k} \cdot \vec{u}_{\vec{k}}$, and $\hat{k}=\vec{k} /|\vec{k}|$ is the unit vector in the direction of $\vec{k}$. Using these fields, we can finally define the longitudinal and transverse correlation functions

$$
\begin{aligned}
S_{L}(k) & =\frac{k^{2}}{N}\left\langle u_{L, \vec{k}} u_{L,-\vec{k}}\right\rangle, \\
S_{T}(k) & =\frac{k^{2}}{N}\left\langle\vec{u}_{T, \vec{k}} \cdot \vec{u}_{T,-\vec{k}}\right\rangle .
\end{aligned}
$$

Following again the approach of conventional elasticity theory, we now have the following expressions [30]

$$
\begin{aligned}
S_{L}(k) & =\frac{\rho T}{B+\frac{4}{3} G} \\
S_{T}(k) & =\frac{\rho T}{G}
\end{aligned}
$$

These expressions directly show that we can calculate the shear modulus $G$ from the low-wavector limit of $S_{T}(k)$ [4]. It is the approach that has been employed to obtain the data shown in Fig. 1 in Sec. III.

Before using these definitions we wish to comment that the various structure factors defined in this section correspond to different mathematical ways of decomposing the particle positions into an average and a fluctuating contributions. In the first approach, we performed the decomposition in the Fourier domain, whereas the second approach deals with position space. It should therefore come as no surprise that the longitudinal part $S_{L}(k)$ can be related to the fluctuating part of the static structure factor $S_{\delta}(k)$. In terms of the particle displacements $\vec{u}_{i}$, the fluctuating part of the density field can be expressed as

$$
\delta \rho_{\vec{k}}=\sum_{j}\left(-i \vec{k} \cdot \vec{u}_{j}+\mathcal{O}\left(k^{2}\right)\right) \exp \left(-i \vec{k} \cdot\left\langle\vec{R}_{j}\right\rangle\right)
$$

Comparing this expression to Eq. (19), we get $\delta \rho_{\vec{k}} \approx$ $-i k u_{L, \vec{k}}$ at the lowest order in $k$. Therefore we conclude that at this order

$$
S_{L}(k) \approx S_{\delta}(k)
$$

and both approaches actually carry equivalent physical content, as they should. By a similar reasoning, we find that

$$
\left\langle\rho_{\vec{k}}\right\rangle=\sum_{j} \exp \left(-i \vec{k} \cdot\left\langle\vec{R}_{j}\right\rangle\right)\left(1-k^{2}\left\langle\left(\hat{k} \cdot \vec{u}_{j}\right)^{2}\right\rangle / 2+\cdots\right)
$$

which shows that an accurate estimate of the configurational part $S_{0}(k)$ can be obtained by computing the structure factor of the average particle positions, a method that could prove convenient for experiments using particle imaging. 
(a)

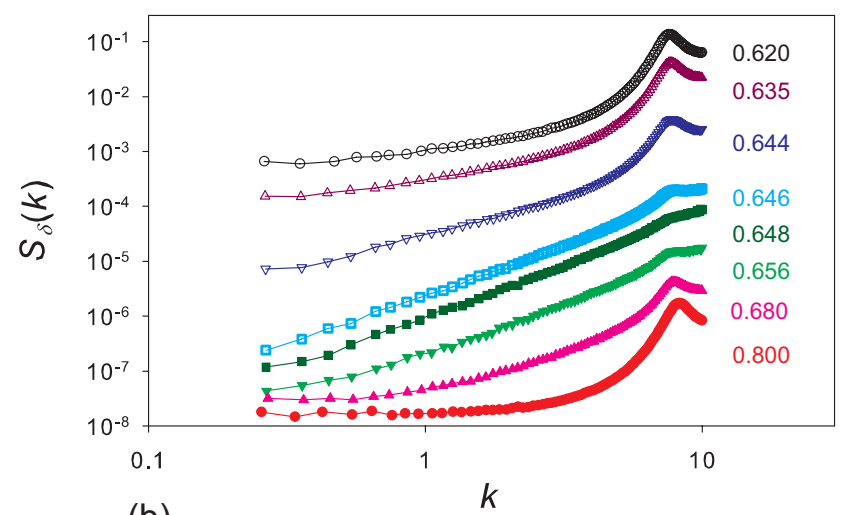

(b)

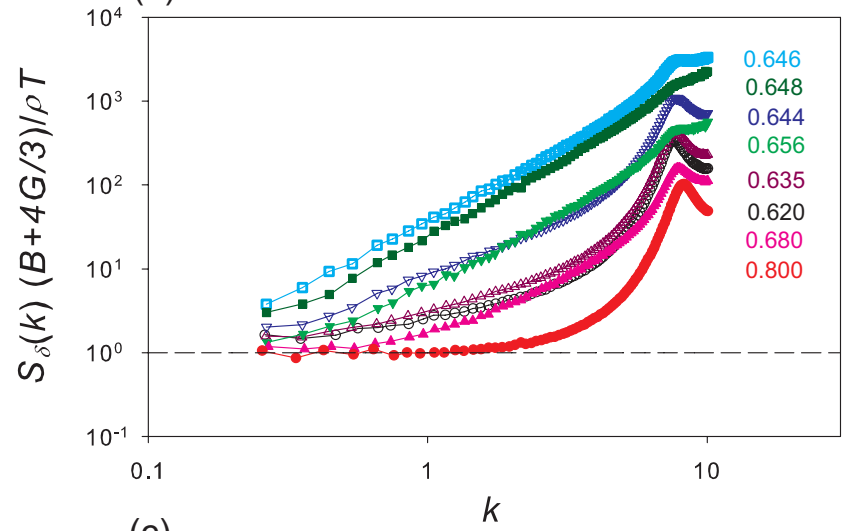

(c)

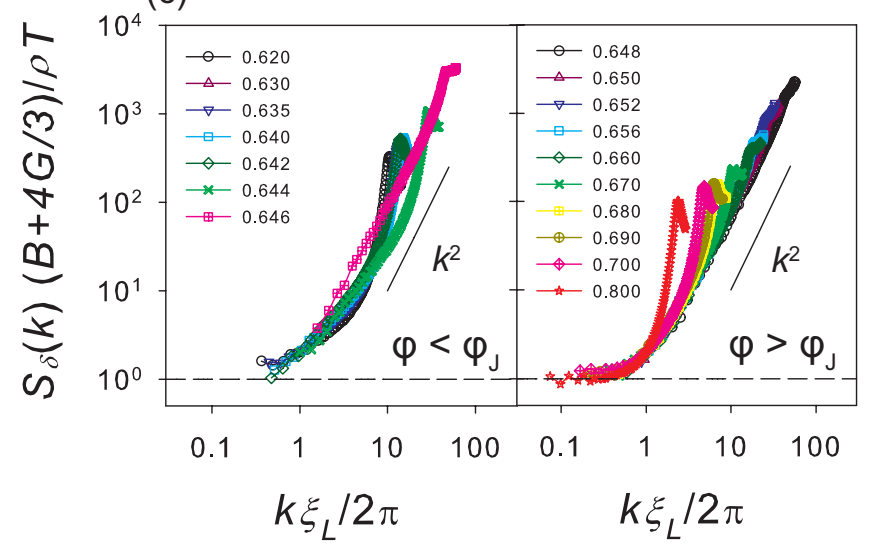

FIG. 2: (Color online) (a) Fluctuation part of the static structure factor $S_{\delta}(k)$ at $T=10^{-8}$ for various densities. (b) Same data with vertical axis scaled with the factor $\left(B+\frac{4}{3} G\right) /(\rho T)$ expected from the usual plane-wave behavior. (c) The horizontal axis is also rescaled to obtain the best data collapse and extract the longitudinal length scale $\xi_{L}$, Eq. (27).

\section{B. Longitudinal fluctuations}

We first discuss the behavior of $S_{\delta}(k)$ in connection with the bulk modulus. In practice, we have calculated $S_{\delta}(k)$ in the following way. We first calculate the density field $\rho_{\vec{k}}$ for each instantaneous configurations of particles, at the lattice points of the first Brillouin zone, $\vec{k}=\left(k_{x}, k_{y}, k_{z}\right)$ in $k$-space where $k_{\alpha}=n_{\alpha} \pi / L$ for $\alpha=x, y, z$ and $n_{\alpha}$ an integer. We then perform a time average to obtain $\left\langle\rho_{\vec{k}}\right\rangle$ for each Fourier component. Using $\rho_{\vec{k}}$ and $\left\langle\rho_{\vec{k}}\right\rangle$ we calculate $\delta \rho_{\vec{k}}$ and obtain $S_{\delta}(\vec{k})$ in a straightforward manner. We finally perform a circular average to obtain the desired $S_{\delta}(k)$.

We plot the numerical results for $S_{\delta}(k)$ at $T=10^{-8}$ and various densities in Fig. 2. The overall amplitude of $S_{\delta}(k)$ strongly decreases when the system is compressed. This is expected, because particles move less and less when density is increased [11], and the overall amplitude of the fluctuations gets smaller. At all densities, we also find that $S_{\delta}(k)$ has a well-defined $k \rightarrow 0$ limit, and that it increases strongly with $k$, with a well-defined first diffraction peak corresponding to the interparticle distance at $k \approx k_{0} \equiv 2 \pi / \sigma$, which reflects the liquid-like structure of amorphous packings at the particle scale.

Two useful informations are contained in these structure factors. We first concentrate on the $k \rightarrow 0$ limits, where the relation Eq. (18) derived from continuum theory is expected to become valid,

$$
\lim _{k \rightarrow 0} S_{\delta}(k)=\frac{\rho T}{B+\frac{4}{3} G} .
$$

To verify this fluctuation formula, we use this expression and replot the same data in a scaled form, observing the $k$-dependence of the quantity $S_{\delta}(k)\left(B+\frac{4}{3} G\right) /(\rho T)$, as shown in Fig. 2 (middle). The bulk and shear moduli are obtained from independent measurements, shown in Fig. 1. Note that since our systems are characterized by large $B / G$ ratio, as discussed in Sec. III it means that the bulk modulus gives the major contribution to the $\left(B+\frac{4}{3} G\right)$ factors in all these expresssions, and the term $G$ is almost always negligible.

The numerical results show that the quantity $S_{\delta}(k)\left(B+\frac{4}{3} G\right) /(\rho T)$ clearly approaches unity as $k \rightarrow 0$, as it should. This observation implies that standard equilibrium relations between the mechanical moduli and static structure factors are valid at large lengthscales for jammed packings, provided the appropriate fluctuating part of the density fields are analysed, instead of the total $S(k)$.

The rescaled plot shows moreover that $S_{\delta}(k)$ not only contains useful information at $k \rightarrow 0$, but that its finite $k$ behaviour is also relevant. The conventional elasticity expression in Eq. (18) does not provide any useful $k$-dependence. In other words, when all the longitudinal waves are the plane waves predicted by conventional elasticity theory, the quantity $S_{\delta}(k)\left(B+\frac{4}{3} G\right) /(\rho T)$ should be unity. Thus, deviations from unity can be interpreted as an indicator for the breakdown of the description by the usual plane wave with the above dispersion relation. Interestingly we find that the rescaled data in Fig. 2 (middle) show finite- $k$ deviations that depend strongly on the density, and are maximal at the jamming density, so that the deviations from conventional elasticity have a remarkable non-monotonic density dependence. Interestingly, near the jamming density, $\varphi \approx \varphi_{J}$, we observe a very clear power law behaviour, $S_{\delta}(k) \propto k^{2}$. 
To characterize quantitatively these deviations and their apparent relation with the jamming criticality, we propose the following scaling analysis of these data. The above description suggests the existence of a non-trivial correlation lengthscale, $\xi_{L}$, separating two distinct behaviours: $S_{\delta}\left(k \xi_{L} \ll 1\right)\left(B+\frac{4}{3} G\right) /(\rho T) \approx 1$, and $S_{\delta}(1 \ll$ $\left.k \xi_{L} \ll k_{0} \xi_{L}\right)\left(B+\frac{4}{3} G\right) /(\rho T) \propto k^{2}$. Therefore, we determined numerically the longitudinal correlation lengthscale $\xi_{L}$ assuming the scaling form

$$
S_{\delta}(k) \approx \frac{\rho T}{B+\frac{4}{3} G} F\left(k \xi_{L}\right),
$$

where $F(x)$ is a scaling function of the form $F(x \ll 1)=$ 1 and $F(x \gg 1) \propto x^{2}$. Physically, this expression implies that $\xi_{L}$ is a characteristic length scale below which the conventional elasticity description of longitudinal particle displacements breaks down. A diverging correlation length $\xi_{L}$ implies that the usual plane wave description does not apply at any length scale.

The results of this scaling analysis are shown in Fig. 2 (bottom). The data collapse is acceptable, but it is difficult to confirm its validity, because the obtained lengthscale $\xi_{L}$ is quite large, and a larger system size would be required for a more accurate determination of this quantity, especially close to $\varphi_{J}$ at very low temperatures. The evolution of the obtained longitudinal lengthscale $\xi_{L}$ with $\varphi$ and $T$ is discussed below in Sec. IVD.

\section{Transverse fluctuations}

We now repeat the analysis of Sec. IVB for the evolution of $S_{T}(k)$ in connection with the shear modulus. We plot $S_{T}(k)$ at $T=10^{-8}$ and various densities in Fig. 3 (top). As for $S_{\delta}(k)$, we find that the overall amplitude of $S_{T}(k)$ decreases rapidly with the density, with an overall wavevector dependence similar to the one of $S_{\delta}(k)$.

We now use the fluctuation formula for the transverse fluctuations 44]

$$
\lim _{k \rightarrow 0} S_{T}(k)=\frac{\rho T}{G}
$$

which is the method we have employed to measure the shear modulus presented in Fig. 1. Therefore, by construction, when we replot the quantity $S_{T}(k)(G / \rho T)$ in Fig. 2 (middle), the data extrapolate to unity when $k \rightarrow 0$.

This figure shows again that the deviations from conventional elasticity have a striking non-monotonic density dependence, and that deviations are maximal when $\varphi$ is close to $\varphi_{J}$. Following the analysis of longitudinal fluctuations, we again hypothesize a scaling behavior for $S_{T}(k)$ :

$$
S_{T}(k) \approx \frac{\rho T}{G} H\left(k \xi_{T}\right)
$$

where $H(x)$ is a scaling function of the form $H(x \ll$ $1)=1$ and $H(x \gg 1) \propto x^{2}$. We show in Fig. 3 (bottom) (a)

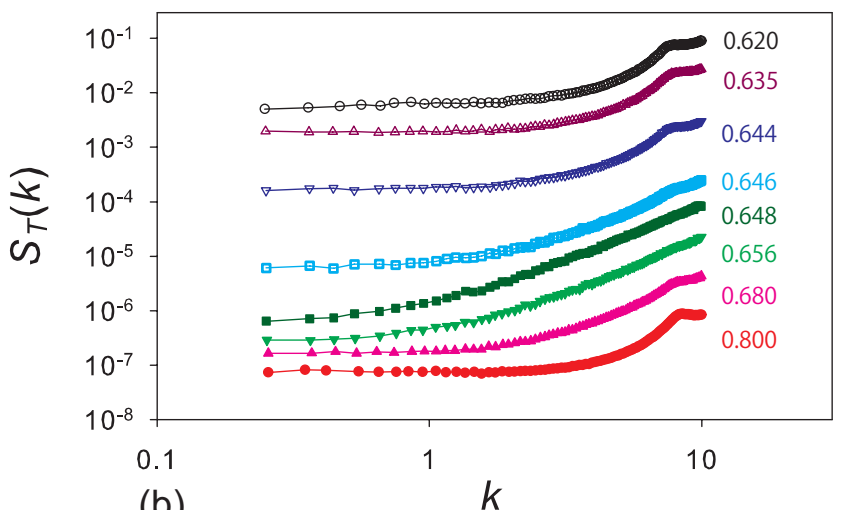

(b)

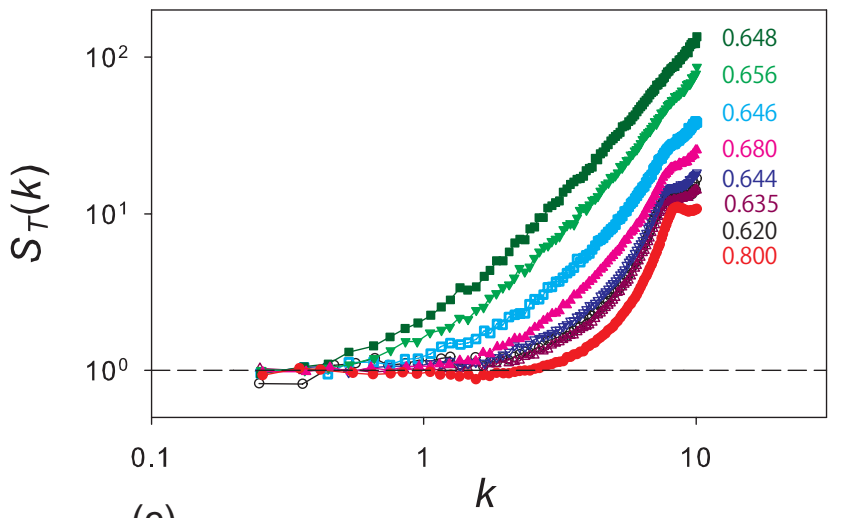

(c)

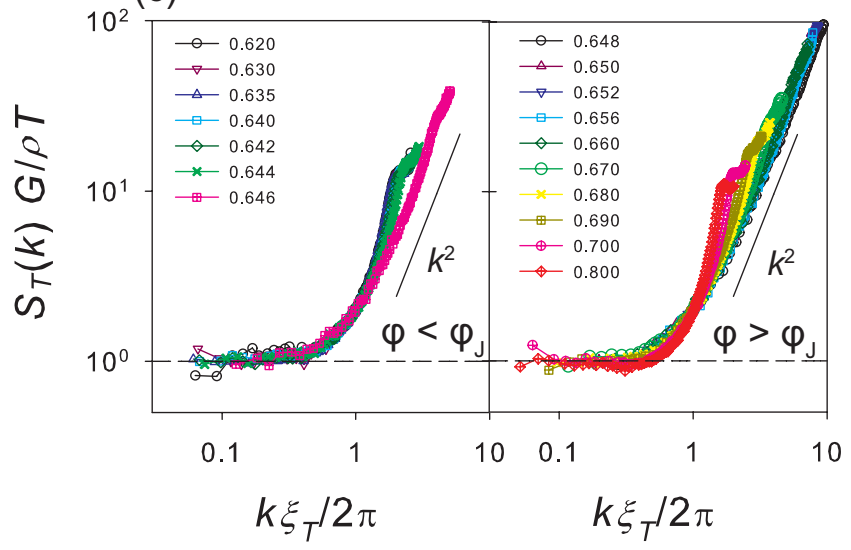

FIG. 3: (Color online) (a) Transverse part of the displacement structure factor $S_{T}(k)$ at $T=10^{-8}$ for various densities. (b) Same data with vertical axis scaled with the factor $G /(\rho T)$ expected from the usual plane-wave behavior. (c) The horizontal axis is also rescaled to obtain the best data collapse and extract the transverse length scale $\xi_{T}$, Eq. (29).

a collapse of the numerical data according to Eq. (29) which allows us to determine numerically a lengthscale $\xi_{T}$ which delimits the validity of the usual plane wave description of transverse fluctuations of the particle displacements. This critical scaling law implies again that the usual plane wave description does not apply at any length scale when $\xi_{T}$ diverges, which is expected exactly at the jamming transition. 

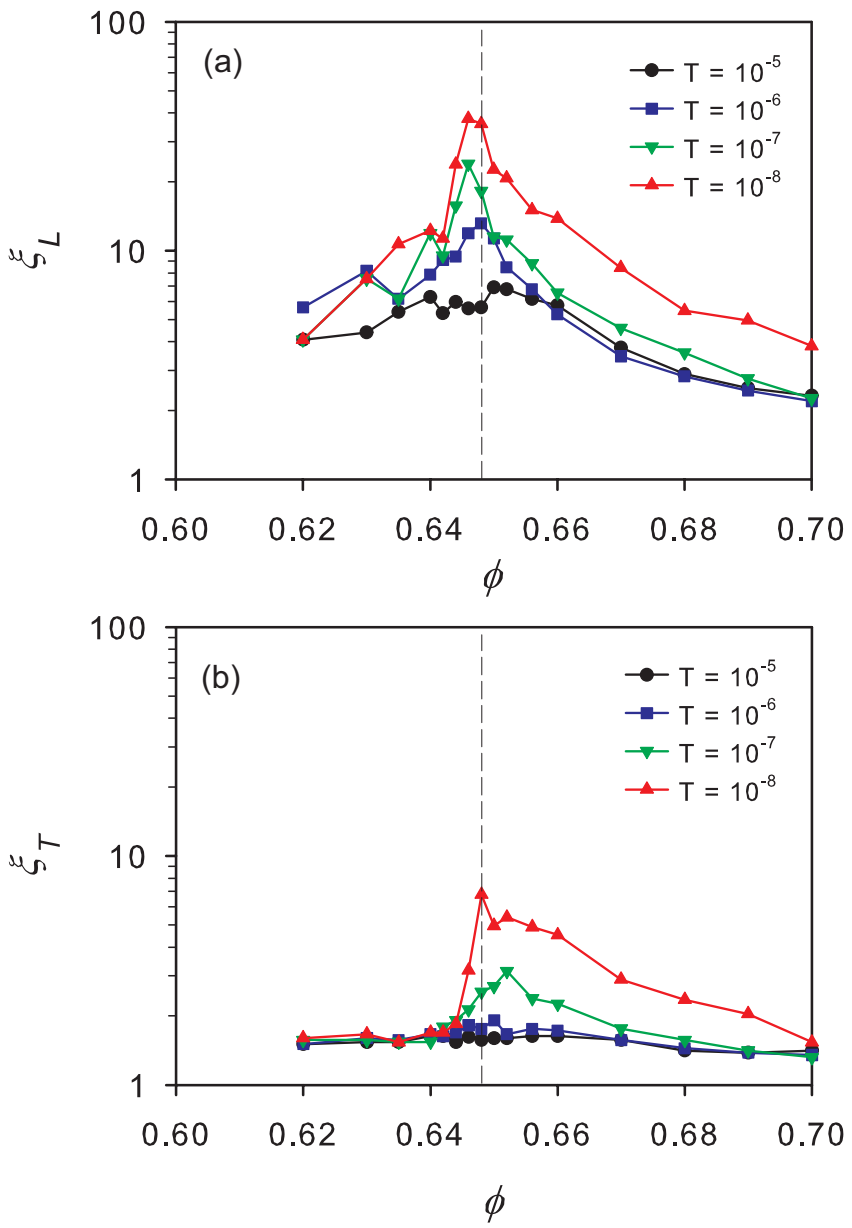

FIG. 4: (Color online) Density dependence of $\xi_{L}$ (a) and $\xi_{T}$ (b) for various temperatures. The near-critical nonmonotonic density dependence observed for $T=10^{-8}$ becomes a smooth variation for $T=10^{-5}$ when jamming criticality is smeared by thermal fluctuations. The usual plane wave description of vibrational motion is therefore excellent away from criticality, $T \gtrsim 10^{-6}$ and/or $\left|\varphi-\varphi_{J}\right| \gtrsim 0.02$.

\section{Transverse and longitudinal lengthscales}

We have performed the scaling analysis outlined in Secs. IVB and IV C for different temperatures and have been able to extract the density and temperature dependences of the characteristic length scales $\xi_{L}$ and $\xi_{T}$. The results are presented in Fig. 4.

Both lengthscales behave qualitatively similarly. At $T=10^{-8}$, they strongly depend on the density: they have a clear maximum for $\varphi \approx \varphi_{J}$ and become of order unity far away from the jamming transition. In particular, we observe that $\xi_{L}$ becomes comparable to the system size $(L=36.2)$ for $T=10^{-8}$, which explains why its numerical determination is difficult. By contrast, the maximum value reached by $\xi_{T}$ is more modest (or order $\xi_{T} \approx 10$ ), explaining why the data collapse for $S_{T}(k)$ is more convincing than the one for $S_{\delta}(k)$.

When the temperature is increased, the density max- imum observed for the correlation lengthscales is much less pronounced, and nearly disappears when $T=10^{-5}$ where $\xi_{L}$ and $\xi_{T}$ have uninteresting density dependences and remain microscopic, $\xi_{L, T} \approx 2-5$. The conclusion is that in this regime, which is relevant for a number of experimental situations for colloidal systems, continuum mechanics actually represents an excellent approximation down to microscopic length scales. In other words, "anomalous" or "soft" modes, which exist over arbitrary length and frequency scales at the jamming transition where correlation lengthscales and timescales are infinite, are strongly suppressed by moving away from jamming criticality.

We now compare our measurements of the lengthscales $\xi_{L}$ and $\xi_{T}$ to similar lengthscales measured earlier in the literature. A first relevant comparison is with the results in Refs. [56, 57] where a characteristic length scale for longitudinal and transverse plane waves at a specific frequency $\omega^{*}=\omega^{*}(\varphi)$ for $\varphi>\varphi_{J}$ and $T=0$ were measured. In this approach, $\omega^{*}$ is a characteristic frequency where the vibrational density of state exhibits anomalous (nearly frequency-independent) behavior [7, 8]. The obtained longitudinal and transverse length scales $\xi_{L}^{*}$ and $\xi_{T}^{*}$ measured from this protocol are predicted to diverge as $\xi_{L}^{*} \propto\left(\varphi-\varphi_{J}\right)^{-0.5}$ and $\xi_{T}^{*} \propto\left(\varphi-\varphi_{J}\right)^{-0.25}$ [56], and the latter behavior is directly confirmed by numerical measurements (data for $\xi_{L}^{*}$ were not shown).

Although it is tempting to assume that $\xi_{L}$ and $\xi_{T}$ have similar physical content as $\xi_{L}^{*}$ and $\xi_{T}^{*}$, power law fits to our measurements yield exponents that are not consistent with the predicted 0.5 and 0.25 (we find that 0.7 and 0.5 fit our data better). However, the lengthscales observed in our measurement is so large that their precise determination is challenging. Much larger systems are needed to fully settle this issue. Furthermore, our lack of knowledge about the precise form of the scaling functions $F(x)$ and $H(x)$ may also affect the quality of our estimates for these lengthscales. We wish to raise the possibity that the two sets of lengthscales are not fully equivalent because we directly defined characteristic lengthscales where the usual plane wave descriptions stop working, whereas Silbert et al. focused on a specific, density-dependent frequency $\omega^{*}[56,57]$.

In a more recent work, Wang et al. [58] have determined numerically the so-called Ioffe-Regel frequencies and lengthscales. These are respectively defined as the timescales and lengthscales characterizing the disappearance of plane waves in the collective dynamic structure factors. We may expect that the lengthscales $\xi_{L}$ and $\xi_{T}$ that we have defined above carry a similar physical content to the Ioffe-Regel lengthscales $l_{L}$ and $l_{T}$ analysed in Ref. [58]. Although some of the numerical results of Wang et al. are consistent with ours, their final conclusions differ qualitatively from ours. In particular, their analysis suggests that in the low-temperature limit $l_{T}$ diverges at $\varphi=\varphi_{J}$ and transverse plane waves do not exist in the hard sphere regime $\varphi<\varphi_{J}$ and $T \rightarrow 0$. Instead, we observe that $\xi_{T}$ becomes finite on both sides of the 


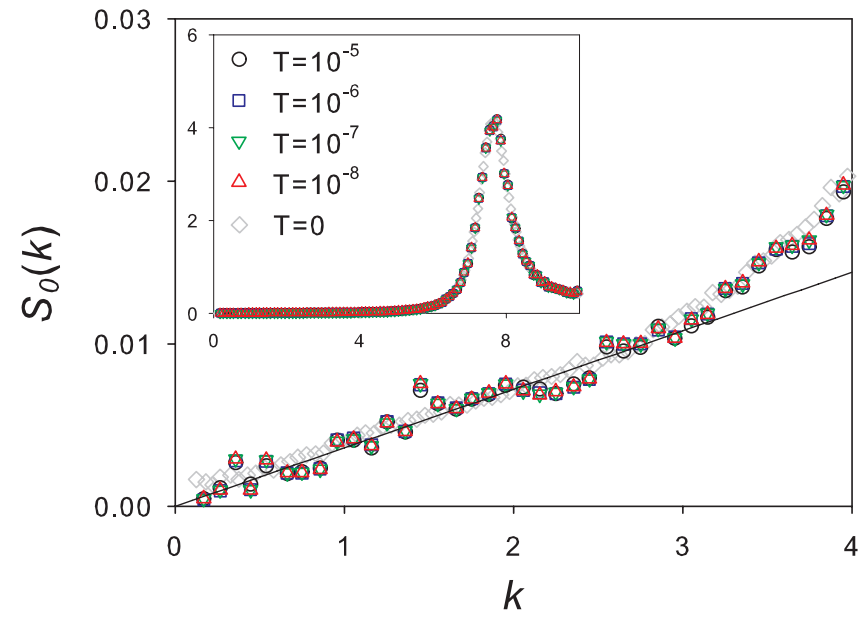

FIG. 5: (Color online) Configurational part of the static structure factor $S_{0}(k)$ at $\varphi=0.652>\varphi_{J}$ and several temperatures. Circles, squares, and upper and lower triangles are represent the data obtained at finite temperatures for $N=64,000$, diamonds indicate data obtained at $T=0$ for $N=512,000$. Solid line indicates the hyperuniform behaviour of density fluctuations at large scale, $S_{0}(k) \propto k$. The hyperuniformity appears essentially independent of temperature. The inset is the zoom out of the main panel.

jamming transition. We suspect that their interpretation is incorrect and stems from extrapolating numerical measurements performed at $\varphi>\varphi_{J}$ to the hard sphere glass. Our results show instead that longitudinal and transverse vibrations do exist in the hard sphere regime, and the associated lengthscales and timescales actually become microscopic as the density is decreased away from $\varphi_{J}$. Contrary to the claim in Ref. [58], the hard sphere glass is not qualitatively different from other amorphous materials.

\section{HYPERUNIFORMITY OF THE CONFIGURATIONAL STRUCTURE FACTOR}

In this section, we analyse the structure factor associated to averaged particle positions and show that this configurational part $S_{0}(k)$ reveals a nearly-hyperuniform behaviour at large length scales. We also show how the analysis of $S_{0}(k)$ allows us to elucidate the physical content of the related nonequilibrium index and nonequilibrium lengthscale measured in amorphous materials.

\section{A. Hyperuniformity is unrelated to jamming criticality}

In Sec. IV A, we decomposed the structure factor $S(k)$ into a fluctuating part, analysed in Sec. IVB, and a configurational part $S_{0}(k)$, which is the subject of the present section.
The first question we ask is whether the configurational part $S_{0}(k)$ is sensitive to the temperature. In Fig. 5], we show numerical results for $S_{0}(k)$ at a constant density $\varphi=0.652$ (slightly above $\varphi_{J}$ ) at several temperatures from $T=10^{-8}$ up to $T=10^{-5}$. The inset shows that $S_{0}(k)$ has the usual wavevector dependence of a liquid structure factor, with a broad first diffraction peak near $k \approx k_{0}$. Clearly, the temperature dependence appears negligible in this representation.

In the main panel, we zoom on the low-wavevector behaviour in order to reveal a possible effect of the thermal fluctuations. Within the accuracy of these computations, we find again no visible temperature dependence for $S_{0}(k)$. This clearly confirms that thermal fluctuations mainly contribute to the fluctuating part of the density fluctuations, whereas the averaged component of density fluctuations is essentially unaffected by the temperature, at least for the range of wavevectors shown in Fig. 5 . We expect more changes to occur at very large wavevectors, $k \gg k_{0}$, where the sharp features associated to the pair correlation function at contact produce long-ranged oscillations [59].

An interesting behaviour is observed for the low- $k$ behaviour of $S_{0}(k)$ in Fig. 5. In the linear scale chosen for representating these numerical measurements, we observe that $S_{0}(k) \propto k$, for $k \lesssim 2$. This "anomalous" linear behavior with an apparent vanishing of $S_{0}(k \rightarrow 0)$ has been termed hyperuniformity [22, 23]. Hyperuniform density fluctuations have been reported in simulations of sphere packings at the jamming transition both numerically 22, 24 26] and in experimentally-constructed granular packings [25]. In colloidal systems, signs of hyperuniformity are much weaker [27 29].

The important conclusion that we can draw from the absence of temperature dependence in the data shown in Fig. [5] is that hyperuniformity appears extremely robust against thermal fluctuations, and can in fact easily be observed even for our highest studied temperature $T=10^{-5}$. This observation is in striking contrast with all other observations reported earlier in this paper related to the jamming criticality. Whereas quantities such as mechanical moduli and correlation lengthscales are rapidly smeared out by thermal fluctuations, hyperuniformity appears rather insensitive to temperature. This strongly suggests that jamming criticality and hyperuniformity are unrelated concepts and have distinct physical origins.

We emphasize that the decomposition of the structure factor $S(k)$ as the sum of two terms $S_{0}(k)$ and $S_{\delta}(k)$ indicates that the total structure factor is related to the isothermal compressibility only through $S_{\delta}(k)$ whose wavevector dependence shows no anomalous dependence, see Fig. 2. On the other hand, we find that $S_{0}(k)$ is characterized by a hyperuniform linear behaviour at low wavevector, but this configurational contribution is unrelated to the compressibility. Thus, we conclude that hyperuniformity (related to $S_{0}$ ) cannot be a logical consequence of a vanishing compressibility (related to $S_{\delta}$ ) of 
the packing. Of course, to observe hyperuniformity in the total structure factor $S(k)$ rather than in the configurational part $S_{0}(k)$, it is necessary that $S_{\delta}(k) \ll S_{0}(k)$, which happens when the compressibility becomes very small. Working close to the jamming transition is therefore a practical rather than a fundamental issue, which is no longer needed when working directly with $S_{0}(k)$. In that sense, the discovery that some amorphous materials become hyperuniform very close to the jamming transition may appear coincidental [22].

These findings are experimentally relevant, because they mean that hyperuniformity, unlike jamming criticality, can actually be observed across a large region of the $(T, \varphi)$ phase diagram for soft colloids. Quite surprisingly, the experimental literature seems to suggest exactly the opposite since a number of experiments have reported signatures of jamming criticality in soft colloids [18, 21] (which, we argue, are actually taken far from criticality), whereas only weak signs of hyperuniformity have been reported [27 29] (which, we argue, should be easily observable). This might be due to the difficult experimental constraint that measuring $S_{0}(k)$ at low $k$ requires detecting the position of a large number of particles with a large precision.

\section{B. Density dependence and deviations from strict hyperuniformity}

Having established that temperature does not affect much the observation of hyperuniformity, we then discuss the effect of the density by analysing data obtained directly at $T=0$. This approach is useful, because it allows us to disentangle temperature and density effects. In addition, we can study at $T=0$ much larger systems in order to analyse whether hyperuniform behaviour can be observed over arbitrarily large lengthscales. To this end, we employ a distinct measurement technique of $S_{0}(k)$ at $T=0$ with a larger number of particles, $N=512,000$, and use 8 independent packings to reduce the statistical error. Our numerical methodology was described in Sec. [II]

Using this larger systems at $T=0$, we confirm in Fig. [5 that $S_{0}(k)$ for this density is in excellent agreement with the finite temperature results obtained with the smaller packings, although of course the statistical error is greatly reduced. The agreement between these two independent sets of data confirms that $S_{0}(k)$ is largely independent of temperature.

Having shown that temperature plays no role, we can now analyse the density dependence of these observations. In Fig. 6] we plot $S_{0}(k)$ measured at $T=0$ at various densities between $\varphi=0.80$ much above jamming, down to $\varphi=0.652$ just above $\varphi_{J}$. We now use a $\log$ - $\log$ representation of the results. At $\varphi=0.80$, $S_{0}(k)$ is almost constant over a large range of wavevectors, $S_{0}(k) \approx 4 \cdot 10^{-3}$ for $k \lesssim 1$, and a hyperuniform behaviour cannot be observed. This behaviour of

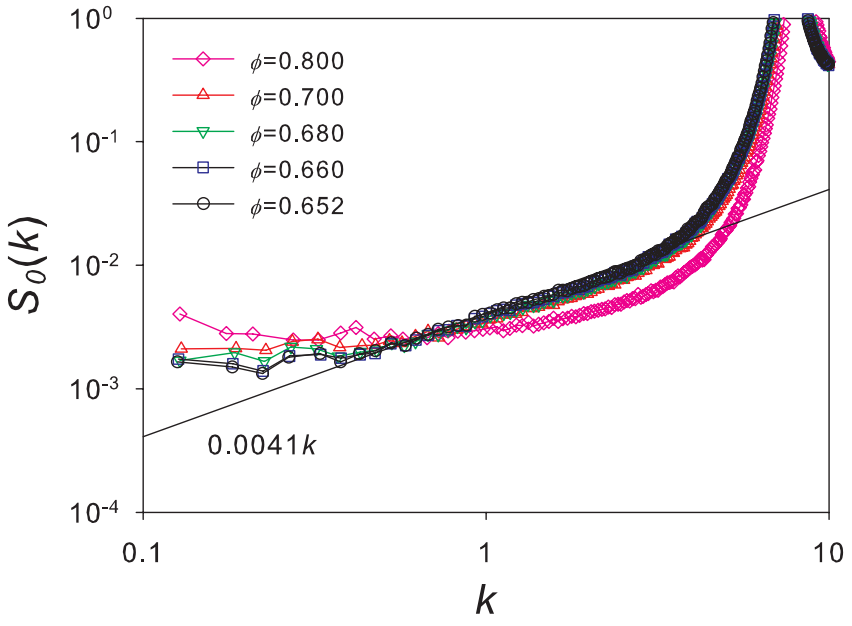

FIG. 6: (Color online) Evolution of $S_{0}(k)$ with the density above the jamming transition in a log-log representation. These data are obtained by averaging over 8 independent packings with $N=512,000$ particles. A clear hyperuniform linear $k$-dependence (shown as a full line) is obtained over a broad range of wavevectors when $\varphi \lesssim 0.7$ with only weak density dependence, but the data saturate to a finite value as $k \rightarrow 0$.

over-compressed packings is consistent with observations made in other glass-forming materials, such as simple Lennard-Jones glasses, where hyperuniformity is not observed either [26].

For $\varphi=0.7$, a linear behavior, $S_{0}(k) \approx 0.004 k$, already appears in wide $k$ region, even though $\left|\varphi-\varphi_{J}\right| \approx 0.055$ is still quite large. More surprising is the observation that the data for $\varphi=0.652,0.66$ and 0.68 (respectively corresponding to $\left|\varphi-\varphi_{J}\right| \approx 0.0063,0.015$, and 0.034 ) are essentially the same, and are characterized by a broad range of wavevectors with linear dependence, $S_{0}(k) \approx 0.004 k$, although the data saturate at very low $k$ to a finite value $S_{0}(k \rightarrow 0) \approx 1.4 \cdot 10^{-3}$. These results indicate that hyperuniformity is a robust feature of $S_{0}(k)$, in the sense that it is weakly dependent on the density and does not require fine-tuning the volume fraction to the jamming density $\varphi_{J}$, confirming that the two concepts are distinct.

However, it should also be noted that a strict hyperuniformity $S(k) \propto k$ can not be observed down to arbitrarily small wavevectors, and deviations appear below $k \approx 0.4$, which corresponds to a large lengthscale $\approx 15 \sigma$. This surprising saturation effect has not been reported before, although we notice that previous literature [22, 26] indicates that the smallest $S_{0}(k \rightarrow 0)$ values achieved in computer simulations are always of the order $10^{-3}$ or more, which is consistent with our own results. This saturation would not be observed if the data in Fig. 6 where plotted in a linear scale.

Our analysis shows that this saturation effect is clearly not due to thermal fluctuations (we work at $T=0$ ). This does not stem from sample-to-sample fluctuations either, because all 8 samples show a saturation of simi- 
lar amplitude. Finally, the saturation does not seem to depend on density, at least for $\varphi>\varphi_{J}$. We cannot access the regime $\varphi<\varphi_{J}$ using energy minimization, but we note that a marked density dependence was reported for the structure factor of hard spheres approaching the jamming density from below in Ref. [22]. However, the total structure factor $S(k)$ was measured in that study, which contains a density-dependent contribution associated to the fluctuating part $S_{\delta}(k)$, which vanishes as $\varphi_{J}$ is approached. It would therefore be very interesting to measure directly $S_{0}(k)$ in the hard sphere glass for very large system sizes. We have performed exploratory simulations with moderate system sizes in this regime and find a weak density dependence of $S_{0}(k)$ when $\varphi>0.62$, but larger systems are needed to analyse more finely the behaviour at very small $k$.

Finally, we remark that it is difficult to provide a physical explanation for the existence of the observed deviations from strict hyperuniformity, mainly because there is no deep physical reason to expect perfect hyperuniformity in these systems in the first place. Among possible factors that could be investigated are the role of a finite density of rattlers in the packings and the role of the specific protocol that is used to prepare the particle packings, which both could influence the measured value of $S_{0}(k \rightarrow 0)$. Such studies are beyond the scope of the present work.

\section{Analysis of the "nonequilibrium index"}

We showed in Sec. IVB that the isothermal compressibility is directly related, for solids at thermal equilibrium, to the fluctuating part of the structure factor via Eq. (18). A direct consequence is that the compressibility is thus not related to the total structure factor $S(k)$ via the relation valid for equilibrium fluids, Eq. (14). To quantify the difference between fluid and solid states, the concept of a "nonequilibrium index", $X$, was introduced and studied both for hard sphere glasses [32] and for other types of amorphous materials [33].

We now show that the decomposition provided above for the structure factor allows us to elucidate the physical content of $X$. Using the notations introduced in the present work, the nonequilibrium index $X$ is defined as 32.

$$
X \equiv \lim _{k \rightarrow 0} \frac{S(k) B}{\rho T}-1
$$

By construction, $X=0$ for a fluid at thermal equibrium, see Eq. (14). Since $X$ is defined as the ratio between fluctuations and response functions, its functional form is also reminiscent of the effective temperature and fluctuation-dissipation ratio that characterize the nonequilibrium of aging and driven glasses [34]. The main difference between the two types of quantities is that $X$ refers to static fluctuations, whereas effective

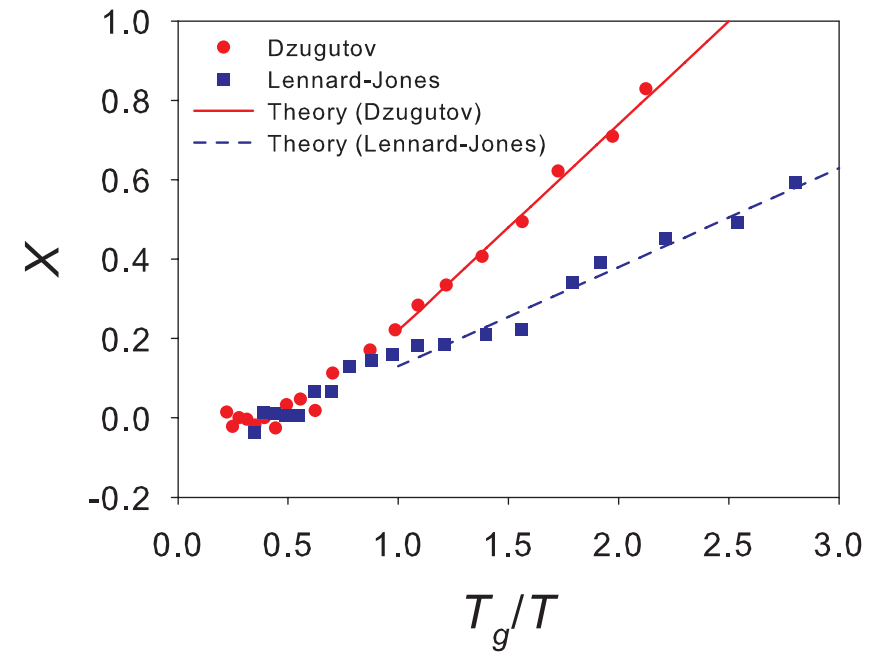

FIG. 7: (Color online) Temperature dependence of the nonequilibrium index $X$ measured in two model glasses (symbols). Full lines are from Eq. (31), the prediction obtained by assuming that equilibrium fluctuation relations are satisfied for the glass.

temperatures are defined from time-dependent correlation and response functions. The non-zero value measured for $X$ in hard sphere glasses was interpreted as a demonstration that the "jammed glassy state is fundamentally nonequilibrium in nature" 32]. The simulations indicate that $X$ grows rapidly when hard sphere glasses are compressed towards $\varphi_{J}$, or when the temperature is decreased below the glass transition temperature $T_{g}$ of model glass-forming systems, such as Lennard-Jones and Dzugutov glasses 33].

The decomposition of the structure factor proposed in Eq. (17) provides us with two important informations. First, we have shown that equilibrium fluctuation relations are perfectly obeyed in the solid phase for static quantities. This result implies that the nonequilibrium nature of the glass cannot be revealed by a fluctuation formula based on static density fluctuations and suggests, in fact, that the introduction of a "nonequilibrium" index to characterize static density fluctuations is unnecessary. This conclusion is in qualitative agreement with the two-temperature scenario for the nonequilibrium dynamics of glasses, where short-time fluctuations and response are typically found to obey equilibrium fluctuation-dissipation relations [34, 35].

Second, the combination of Eqs. (17, 18) provides predictions for the leading behaviour of the nonequilibrium index in various systems. For glass-forming models with continuous interactions, we can assume that $S_{0}(k \rightarrow 0)$ and the bulk modulus are weakly temperature dependent deep in the glass phase [59], so that in the lowtemperature limit, one gets

$$
X\left(T \ll T_{g}\right) \approx \frac{S_{0}(k \rightarrow 0) B}{\rho T} \propto \frac{1}{T} .
$$

In Fig. 7. we confirm that the low-temperature behaviour 

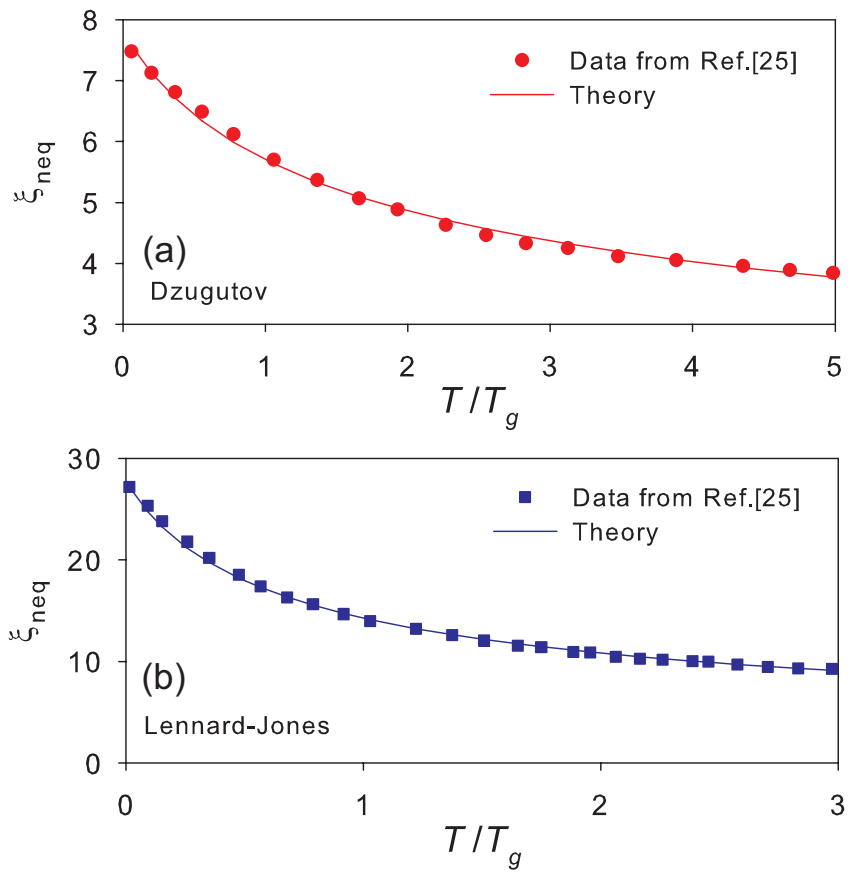

FIG. 8: (Color online) Temperature dependence of the nonequilibrium lengthscale $\xi_{\text {neq }}$ measured in two model glasses (symbols). Full lines are from Eq. (34), the prediction obtained by assuming the equilibrium fluctuation relations are satisfied for the glass.

of the nonequilibrium index measured numerically in Ref. 33] is consistent with our prediction in Eq. (31) that it should diverge as $1 / T$ as $T \rightarrow 0$. This leading temperature behaviour stems from the fact that $S(k)$ in the definition of $X$ in Eq. (30) contains a 'frozen' contribution, $S_{0}$, which does not vanish at $T=0$.

For hard sphere glasses, the leading asymptotic behaviour of the nonequilibrium index depends strongly of the hypothesis made regarding the behaviour of $S_{0}(k)$ very close to $\varphi_{J}$. Assuming that hyperuniformity is only approximate, as we report in Fig. 6] one would then predict that $X$ in Eq. (30) is dominated by the divergence of the bulk modulus, yielding $X \approx\left(\varphi_{J}-\varphi\right)^{-2}$. In Ref. 32. a linear decrease, $S_{0}(k \rightarrow 0) \sim\left(\varphi-\varphi_{J}\right)$, was assumed for $S_{0}$, which turns into a different divergent behaviour, $X \sim\left(\varphi_{J}-\varphi\right)^{-1}$, for the nonequilibrium index. $\mathrm{Nu}-$ merically, we expect that $X$ exhibits a crossover between these two power law regimes as $\varphi_{J}$ is approached, which could be difficult to analyse.

\section{Analysis of the "nonequilibrium lengthscale"}

We finally discuss the concept of a nonequilibrium lengthscale, $\xi_{\text {neq }}$, defined again for amorphous materials from the behaviour of the static structure structure. The nonequilibrium lengthscale is defined as 33]

$$
\xi_{\text {neq }} \equiv[-c(k \rightarrow 0)]^{1 / d} \approx[\rho S(k \rightarrow 0)]^{-1 / d},
$$

where $c(k)$ is the direct correlation function [31] and $d$ is the dimensionality of the system. In the final part of Eq. (32), we have assumed that $S(k \rightarrow 0) \ll 1$. In computer simulations, it is found that $\xi_{\text {neq }}$ grows as temperature is decreased below $T_{g}$ in model glasses [33] and saturates to a finite value as $T \rightarrow 0$, whereas it is predicted to diverge as $\varphi \rightarrow \varphi_{J}$ in hard sphere glasses [32]. As such, it is interpreted as a growing static length scale that is potentially relevant to characterize the structure of the glassy state. In this view, hard sphere glasses would therefore be somewhat "special" since they would have a diverging static lengthscale, whereas glasses with continuous interactions would exhibit a non-diverging static lengthscale.

Because this lengthscale directly follows from the low$k$ behaviour of the structure factor, our decomposition (17) into two distinct contributions is again relevant to understand the physical content of the nonequilibrium lengthscale, which we can rewrite

$$
\xi_{\text {neq }}=\left[\rho S_{0}(k \rightarrow 0)+\frac{\rho^{2}}{B+\frac{4}{3} G} T\right]^{-1 / d} .
$$

For Lennard-Jones and Dzugutov models, we again expect that $S_{0}(k \rightarrow 0)$ and the mechanical moduli are weakly dependent on temperature far below $T_{g}$, so that the leading temperature dependence of the nonequilibrium lengthscale is transparent in Eq. (33), and should be of the form

$$
\xi_{\text {neq }} \approx(a+b T)^{-1 / d}
$$

where $a$ and $b$ are some constants. In Fig. 8, we confirm that this prediction describes the numerical data very well for two glass-formers, showing that the growth of the nonequilibrium lengthscale with decreasing temperature can in fact be fully understood by assuming that density fluctuations obey equilibrium fluctuation formula. In essence, therefore, the growth of the nonequilibrium lengthscale in the glass phase reflects the competition between the configurational and fluctuating parts of the static structure factor, which have different temperature dependences: the former is essentially constant and reflects the 'inherent' structure of the glass, the latter stemming from vibrational motion and is thus proportional to temperature, as captured in Eq. (34).

For hard sphere glasses, the behaviour of the nonequilibrium length would again depend sensitively on the behaviour of $S_{0}(k)$ near $\varphi_{J}$. Assuming that hyperuniformity is only approximate, the nonequilibrium lengthscale would grow strongly as the glass phase is entered and the compressibility decreases, but its growth would saturate to a value $\xi_{\text {neq }} \approx\left[\rho S_{0}(k \rightarrow 0)\right]^{-1 / d} \approx 8.2$, using numerical values from Fig. 6. Interestingly, this saturation value is close to the value $\xi_{\text {neq }}(T \rightarrow 0) \approx 7.5$ found for the three-dimensional Dzugutov glass-former in Fig. 8, which could support the idea that hard sphere glasses are not a "special" type of glass-former. For a strictly hyperuniform hard sphere system, on the other hand, the 
nonequilibrium lengthscale would diverge as $\varphi \rightarrow \varphi_{J}$, as predicted in Ref. [32].

\section{CONCLUSION}

In this work, we have analyzed the density and temperature dependences of mechanical moduli and several types of structure factors in a model system of soft harmonic spheres in the vicinity of the jamming transition.

We have shown that thermal fluctuations very quickly erase several signatures of the criticality associated to jamming, in agreement with earlier work related to single particle dynamics [11]. We showed that the bulk modulus, the shear modulus, the longitudinal and transverse lengthscales rapidly acquire a 'normal' behaviour typical of ordinary solids, whereas the large lengthscales and timescales associated to the isostatic jamming critical point are only observed in a narrow region of the $(T, \varphi)$ phase diagram. We conclude that most colloidal experiments to date have hardly been able to probe the jamming criticality, nor have the thermal vestiges of the jamming transition that result from the existence of nonmicroscopic lengthscales and timescales been observed. These conclusions suggest that the soft and hard sphere glasses that are commonly studied experimentally essentially behave as ordinary solids where the usual plane wave description holds down to small length scales, as concluded from a very recent experimental study [60]. Therefore, we hope that our results will encourage further experimental investigations of these issues, for instance using emulsion droplets [53], or core-shell microgel particles 61.

A second major finding in our study is that density fluctuations for jammed colloidal systems follow the laws of equilibrium thermodynamics and their study does not reveal the nonequilibrium nature of glasses. Our analysis is based on a decomposition of density fluctuations in a configurational and fluctuating parts. Whereas the fluctuating part is directly related to mechanical moduli via equilibrium fluctuation formula, we found that the configurational part is essentially independent of both density and temperature in a rather broad range of parameters. The decomposition into these two components allows us to elucidate the behaviour reported in earlier numerical studies for the nonequilibrium index and nonequilibrium lengthscales characterizing amorphous materials. Based on these observations, we have suggested that hyperuniformity observed in the configurational structure factor is unrelated to the compressibility, and therefore to the jamming criticality.

These results raise some interesting questions. It has been established numerically that the same jamming criticality is observed for packings with very different preparation protocols 62]. Our observation that a strict hyperuniformity is not observed in our packings suggests that the value of $S_{0}(k \rightarrow 0)$ could very well be affected by the nonequilibrium protocol used to prepare packings. One could for instance hypothesize that a packing prepared with a slower annealing could be more hyperuniform than one produced via brutal compression. This raises the appealing possibility that the nonequilibrium lengthscale $\xi_{\text {neq }}$ measured either at $T=0$ (for continuous potentials) or at infinite pressure (for hard spheres) truly encodes some non-trivial information about the glassy state [32]. If correct, it would mean that it is not really the temperature or density dependences of $\xi_{\text {neq }}$ which truly matter, but rather its evolution for different preparation histories. Therefore, we believe that it would be interesting to understand better the physical content of this quantity in various glassy materials prepared using various thermal histories.

\section{Acknowledgments}

We thank S. Teitel for useful discussions, H. Mizuno for helpful comments, and E. Corwin for sharing some of his packings with us. The research leading to these results has received funding from the European Research Council under the European Union's Seventh Framework Programme (FP7/2007-2013) / ERC Grant agreement No 306845, and from JSPS KAKENHI Grant Number 26887021 .
[1] A. J. Liu, S. R. Nagel, W. van Saarloos, and M. wyart in Dynamical Heterogeneities in Glasses, Colloids and Granular Materials, Eds.: L. Berthier, G. Biroli, J.-P. Bouchaud, L. Cipelletti, and W. van Saarloos (Oxford University Press, Oxford, 2011).

[2] M. van Hecke, J. Phys.: Condens. Matter 22, 033101 (2010).

[3] G. Parisi and F. Zamponi, Rev. Mod. Phys. 82, 789 (2010).

[4] C. F. Moukarzel, Phys. Rev. Lett. 81, 1634 (1998).

[5] A. V. Tkachenko and T. A Witten, Phys. Rev. E 60, 687 (1999).

[6] J.-N. Roux, Phys. Rev. E 61, 6802 (2000).
[7] C. S. O'Hern, L. E. Silbert, A. J. Liu, and S. R. Nagel, Phys. Rev. E 68, 011306 (2003).

[8] M. Wyart, L. Silbert, S. R. Nagel, and T. A. Witten, Phys. Rev. E 72, 051306 (2005).

[9] C. Brito and M. Wyart, EPL 76, 149 (2006).

[10] C. Brito and M. Wyart, J. Chem. Phys. 131, 024504 (2009).

[11] A. Ikeda, L. Berthier, and G. Biroli, J. Chem. Phys. 138, 12A507 (2013).

[12] L. Berthier, H. Jacquin, and F. Zamponi, Phys. Rev. E 84, 051103 (2011).

[13] H. Jacquin, L. Berthier, and F. Zamponi, Phys. Rev. Lett. 106, 135702 (2011). 
[14] A. Ikeda, L. Berthier, and P. Sollich, Phys. Rev. Lett. 109, 018301 (2012).

[15] A. Ikeda, L. Berthier, and P. Sollich, Soft Matter 9, 7669 (2013).

[16] E. DeGiuli, E. Lerner, C. Brito, and M. Wyart, Proc. Natl. Acad. Sci. USA 111, 17054 (2014).

[17] E. DeGiuli, E. Lerner, and M. Wyart, arXiv:1501.06995.

[18] Z. Zhang, N. Xu, D. T. N. Chen, P. Yunker, A. M. Alsayed, K. B. Aptowicz, P. Habdas, A. J. Liu, S. R. Nagel, and A. G. Yodh, Nature (London) 459, 230 (2009).

[19] L. Wang and N. Xu, Soft Matter 9, 2475 (2013).

[20] T. Bertrand, C. F. Schreck, C. S. OHern, and M. D. Shattuck, Phys. Rev. E 89, 062203 (2014).

[21] T. Still, C. P. Goodrich, K. Chen, P. J. Yunker, S. Schoenholz, A. J. Liu, and A. G. Yodh Phys. Rev. E 89, 012301 (2014).

[22] A. Donev, F. H. Stillinger, and S. Torquato, Phys. Rev. Lett. 95, 090604 (2005).

[23] S. Torquato and F. H. Stillinger, Phys. Rev. E 68, 041113 (2003).

[24] C. E. Zachary, Y. Jiao, and S. Torquato, Phys. Rev. Lett. 106, 178001 (2011).

[25] L. Berthier, P. Chaudhuri, C. Coulais, O. Dauchot, and P. Sollich, Phys. Rev. Lett. 106, 120601 (2011).

[26] L. E. Silbert and M. Silbert, Phys. Rev. E, 80, 041304 (2009).

[27] R. Kurita and E. R. Weeks, Phys. Rev. E 82, 011403 (2010).

[28] R. Kurita and E. R. Weeks, Phys. Rev. E 84, 030401 (2011).

[29] R. Dreyfus, Y. Xu, T. Still, L. A. Hough, A. G. Yodh, and S. Torquato, Phys. Rev. E 91, 012302 (2015).

[30] P. M. Chaikin and T. C. Lubensky, Principles of Condensed Matter Physics, (Cambridge University Press, Cambridge, 1995).

[31] J. P. Hansen and I. R. McDonald, Theory of Simple Liquids, (Elsevier, Amsterdam, 1986).

[32] A. B. Hopkins, F. H. Stillinger, and S. Torquato, Phys. Rev. E 86, 021505 (2012).

[33] E. Marcotte, F. H. Stillinger, and S. Torquato, J. Chem. Phys. 138, 12A508 (2013).

[34] L. F. Cugliandolo, J. Kurchan, and L. Peliti, Phys. Rev. E 55, 3898 (1997).

[35] J. Kurchan, Nature (London) 433, 222 (2005).

[36] D. J. Durian, Phys. Rev. Lett. 75, 4780 (1995).

[37] P. Olsson and S. Teitel, Phys. Rev. Lett. 99, 178001 (2007).

[38] L. Berthier and T. A. Witten, EPL 86, 10001 (2009).

[39] L. Berthier and T. A. Witten, Phys. Rev. E 80, 021502 (2009).

[40] M. P. Allen and D. J. Tildesley, Computer Simulation of
Liquids, (Oxford University Press, Oxford, 1987).

[41] E. Bitzek, P. Koskinen, F. G'ahler, M. Moseler, and P. Gumbsch, Phys. Rev. Lett. 97, 170201 (2006).

[42] A. Donev, F. H. Stillinger, and S. Torquato, Phys. Rev. E 71, 011105 (2005).

[43] L. Berthier and G. Biroli, Rev. Mod. Phys. 83, 587 (2011).

[44] C. L. Klix, F. Ebert, F. Weysser, M. Fuchs, G. Maret, and P. Keim, Phys. Rev. Lett. 109, 178301 (2012).

[45] S. Dagois-Bohy, B. P. Tighe, J. Simon, S. Henkes, and M. van Hecke, Phys. Rev. Lett. 109, 095703 (2012).

[46] J. P. Wittmer, H. Xu, and J. Baschnagel, Phys. Rev. E 91, 022107 (2015).

[47] P. Charbonneau, J. Kurchan, G. Parisi, P. Urbani and F. Zamponi, Nature Comm. 5, 3725 (2014).

[48] H. Yoshino and F. Zamponi, Phys. Rev. E 90, 022302 (2014).

[49] M. Wyart, Annales de Physique 30, 1 (2005).

[50] W. G. Ellenbroek, Z. Zeravcic, W. van Saarloos, and M. van Hecke, EPL 87, 34004 (2009).

[51] H. Jacquin and L. Berthier, Soft Matter 6, 2970 (2010).

[52] A. Basu, Y. Xu, T. Still, P. E. Arratia, Z. Zhang, K. N. Nordstrom, J. M. Rieser, J. P. Gollub, D. J. Durian, and A. G. Yodh, Soft Matter 10, 3027 (2014).

[53] F. Scheffold, F. Cardinaux, and T. G. Mason, J. Phys.: Condens. Matter 25, 502101 (2013).

[54] G. Szamel and E. Flenner, Phys. Rev. Lett. 107, 105505 (2011).

[55] F. H. Stillinger and T. A. Weber, Phys. Rev. A 25, 978 (1982).

[56] L. E. Silbert, A. J. Liu, and S. R. Nagel, Phys. Rev. Lett. 95, 098301 (2005).

[57] L. E. Silbert, A. J. Liu, and S. R. Nagel, Phys. Rev. E, 79, 021308 (2009).

[58] X. Wang, W. Zheng, L. Wang, and N. Xu, Phys. Rev. Lett. 114, 035502 (2015).

[59] A. Ikeda and L. Berthier, Phys. Rev. E 88, 052305 (2013).

[60] C. L. Klix, G. Maret, and P. Keim, arXiv:1503.08968

[61] J. J. Crassous, L. Casal-Dujat, M. Medebach, M. ObiolsRabasa, R. Vincent, F. Reinhold, V. Boyko, I. Willerich, A. Menzel, C. Moitzi, B. Reck, and P. Schurtenberger, Langmuir 29, 10346 (2013).

[62] P. Chaudhuri, L. Berthier, and S. Sastry, Phys. Rev. Lett. 104, 165701 (2010).

[63] To arrive to this expression, we first evaluate $S_{L}(k)$ using the dispersion relation, and used Eq. (24) to estimate $S_{\delta}(k)$. Details of the evaluation of $S_{L}(k)$ can be found in pp. 321-322 of Ref. 30]. 